\title{
SINGLE INCISION LAPAROSCOPIC MANAGEMENT OF A RUPTURED HETEROTOPIC PREGNANCY PRESENTING AS AN ACUTE APPENDICITIS: A CASE REPORT
}

\author{
Ross O. Downes (Corresponding Author)
}

General Surgery, Laparoscopy

Bsc MB BS DM

Doctors Hospital

Nassau, Bahamas

Tel\# 12423760893

Fax\#-12426760036

Email: rossdownes@yahoo.com

Financial support: No contribution

\section{Learning points:}

* The incidence of heterotopic pregnancies is rising in both assisted and spontaneous pregnancies.

* A high index of suspicion and awareness is required for diagnosis.

* Caution is therefore advised during surgical treatment of ectopic gestation when a coexisting intrauterine pregnancy is present.

MeSH keywords: Laparoscopy, single incision, single port, appendectomy, Heterotopic, ectopic 


\section{INTRODUCTION}

Heterotopic pregnancy, defined as the coexistence of an intrauterine pregnancy and an extrauterine pregnancy. It is a rare entity and theoretically occurs at an incidence of 1 in 30000 pregnancies $(2,3,12)$. The rise in the incidence of tubal and pelvic diseases has contributed to an increasing rate of heterotopic pregnancies. This rise can also be attributed to the increasing use of assisted reproductive technology such as In Vitro Fertilization (IVF) and Gamete intra-fallopian transfer (GIFT) $\quad(2,3,11,12)$. After assisted reproductive technology procedures, the incidence of heterotopic pregnancy is at a concerning 1 in 100 pregnancies $(2,12)$.

The treatment of heterotopic pregnancy aims to maintain the intrauterine pregnancy while removing the extrauterine pregnancy using a minimally invasive method (1). The treatment modalities for heterotopic pregnancy can be divided into surgical and non-surgical treatments. Non-surgical treatments involve the direct administration of drugs, such as potassium chloride, methotrexate, and hyperosmolar glucose to the extrauterine gestational sac under ultrasonographic or laparoscopic guidance $(10,11)$. Systemic management of these agents is difficult because these treatments can result in termination of both the desirable intrauterine and unwanted extrauterine pregnancy (7). Surgical management, particularly laparoscopic surgery, has a shorter operating time, faster recovery, and less postoperative pain compared with those of laparotomy.

As seen in our patient, heterotopic pregnancy is a difficult entity to diagnose, often leading to the late detection of an extrauterine sac (2). Our patient is a 36year-old who has been trying to get pregnant for 2 years. Because of the information available a diagnosis of acute appendicitis in pregnancy was made. There were no clear signs of a ruptured ectopic pregnancy despite ultrasonography been done. An emergency laparoscopic procedure was done highlighting the underlining pathology.

\section{CASE PRESENTATION}

We present a case of heterotopic pregnancy after spontaneous conception. Our patient is a 36-year-old who has been trying to conceive for 2 years. She is a gravida 1 para $1^{+1}$ who has a 4 -year-old boy delivered by normal vaginal delivery previously and also had one previous presentation for a unruptured ectopic with salpingostomy. She presented to the emergency department with a history of abdominal pain of $12 \mathrm{hr}$ duration that was gradual in onset, beginning peri-umbilical then migrating to the right lower quadrant. The pain did not radiate but was associated with nausea and vomiting. She had visited her obstetrician 1-week prior and was noted to be 6 weeks pregnant with an intrauterine gestation confirmed on ultrasonography. She had a positive pregnancy test. She had no vaginal bleeding. Initial examination revealed a well-hydrated patient with blood pressure of 130/84 and pulse rate of $54-90 / \mathrm{min}$. Abdomen was tender in the right lower quadrant and suprapubically with guarding and rebound tenderness. Vaginal examination revealed cervical excitation tenderness and fullness in Right adnexa. Her laboratory results were all normal expect for an elevated WBC of 17.1. She 
had a repeat transvaginal US confirms the live intrauterine pregnancy (marked by the blank arrow) by demonstrating a fetal heart rate of 131 beats / minute on pulse doppler. The image also shows a well-defined relatively thin walled anechoic left ovarian corpus luteal cyst (marked by the solid white arrow) in a deeper plane beyond the uterine contour and non-visualization of the appendix (see below).

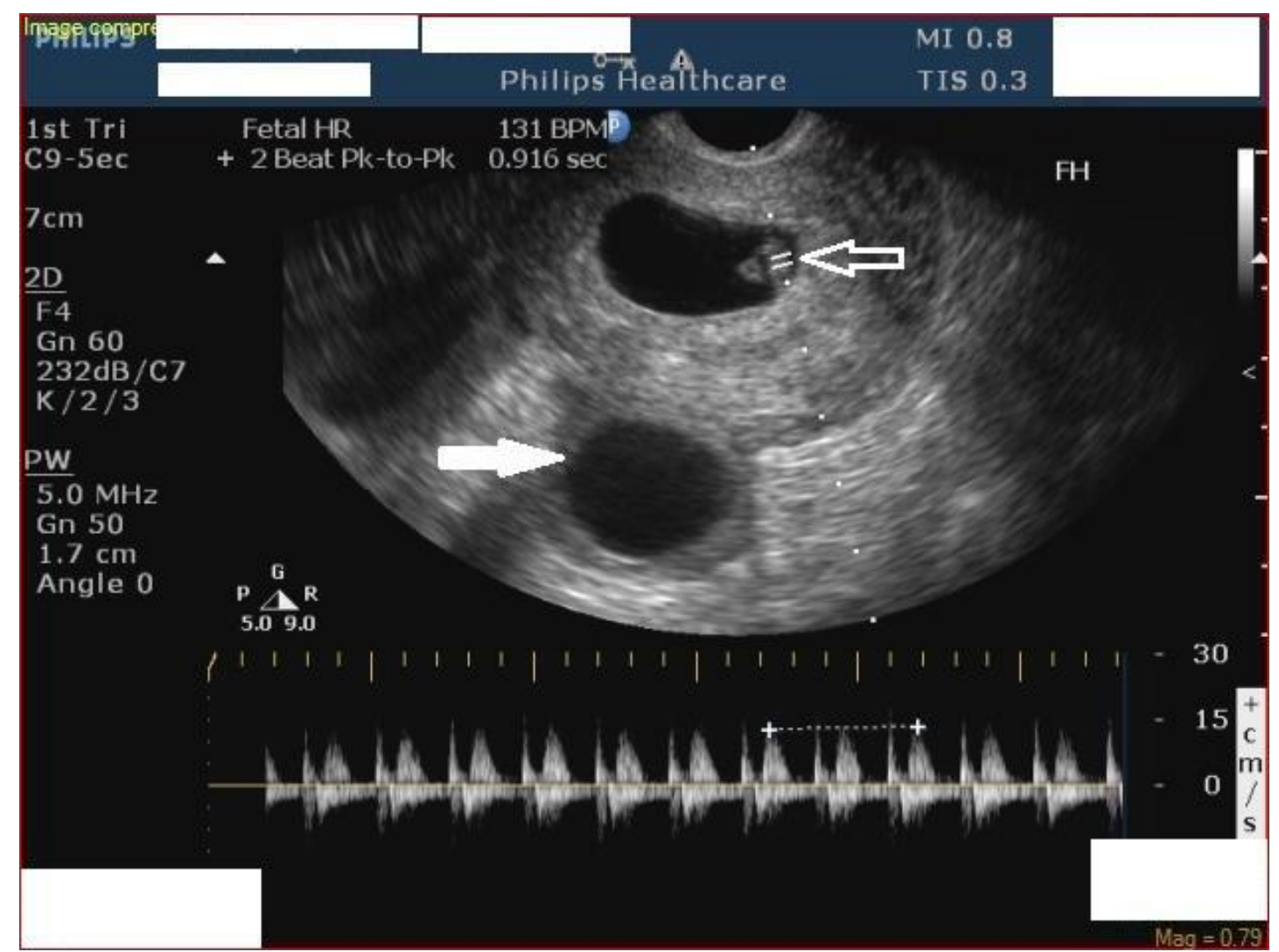

Fig 1 showing live intrauterine pregnancy (blank arrow) and well defined left corpus luteal cyst ( solid arrow).

A clinical diagnosis of acute appendicitis was made and an emergency single port laparoscopic appendectomy was advised. At laparoscopy, via a 2.0-2.5 $\mathrm{cm}$ vertical trans-umbilical skin incision, the peritoneal cavity entered and an Olympus single port Triport platform introduced. After pneumoperitoneum established, a ruptured right isthmic ectopic gestation with $500 \mathrm{ml}$ hemoperitoneum was identified and a salpingectomy performed using LigaSure $^{\mathrm{TM}}$. An appendectomy was also performed. She also had drainage of a large right ovarian cyst. The uterus was not manipulated. Postoperative recovery was uneventful and our initial diagnosis changed to ruptured heterotopic pregnancy. The patient was discharged in 2 days. Histopathological examination confirmed the presence of chorionic villi and ectopic pregnancy in the tube. The patient's intrauterine pregnancy remained viable and went on to have full term caesarian section of a healthy male infant. 


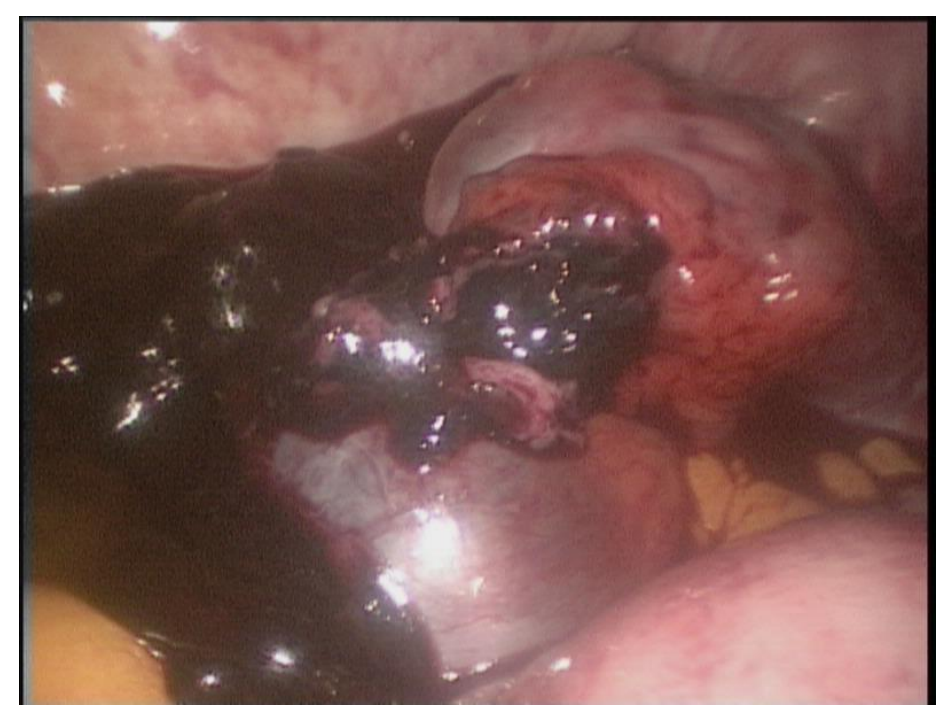

Fig 2 Hemoperitoneum from ruptured heterotopic pregnancy covering ovarian cyst

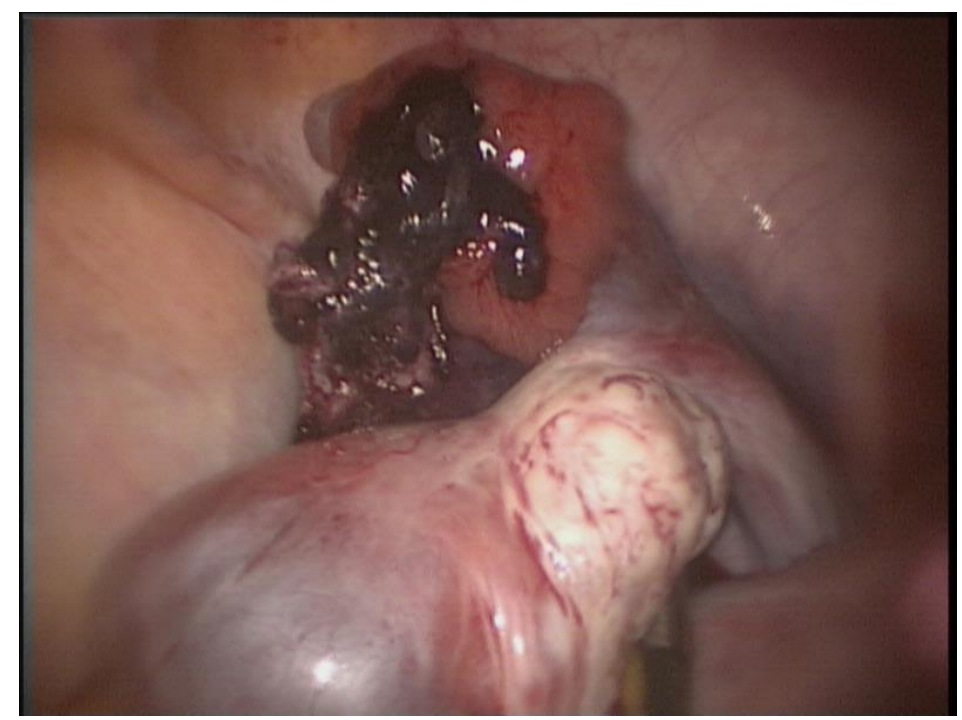

Fig 3 Ruptured fallopian tube and cyst

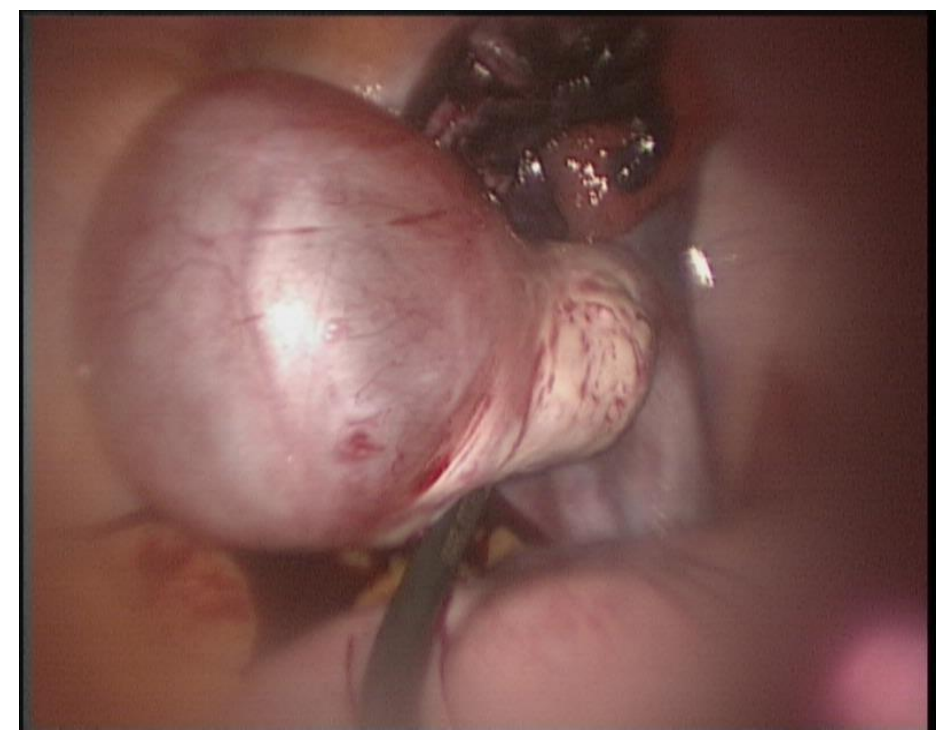

Fig 4 ovarian cyst isolated, ruptured heterotopic pregnancy seen 


\section{DISCUSSION}

Heterotopic pregnancy, defined as the coexistence of an intrauterine pregnancy and an extrauterine pregnancy. Heterotopic pregnancy is often a serious emergency and is frequently associated with life-threatening bleeding in cases of delayed diagnosis (1). It is believed that the incidence of heterotopic pregnancy is under-represented in the literature. It is estimated at an incidence of 1 in 30000 $(2,11,12)$. The natural history is that a significant proportion of ectopic pregnancies undergo spontaneous resolution (6). One series reported a staggering rate of heterotopic pregnancies diagnosed at laparoscopy or laparotomy as high as $74 \%$ (8). This rate is noted to substantially more alarming in patients undergoing assisted reproductive procedures. In that instance, a rate as high as 1 in 300 has been reported $(2,12)$. In the general population, the major risk factors for heterotopic pregnancy are the same as those for ectopic pregnancy. These factors include previous ectopic pregnancy, tubal pathology and surgery, genital infections, intrauterine devices, multiple sexual partners, smoking and maternal age. For women in an assisted reproductive program, additional factors include multiple ovulation, transferring >4 embryos, abnormal tubal anatomy and technical factors related to embryo transfer (13). One review showed $71 \%$ cases had one risk factor and $10 \%$ had three or more risk factors. In the same review, $29 \%$ of cases had no risk factors, thus the role of imaging becomes critical in diagnosis (12).

A high index of suspicion and awareness is required for diagnosis. Ectopic pregnancy should be considered in any woman of childbearing age with abdominal pain and/or abnormal vaginal bleeding. Up to $18 \%$ of women who present with first trimester bleeding will be diagnosed with ectopic pregnancy (6). Differentials for heterotopic pregnancy include normal intrauterine pregnancy, a normal intrauterine pregnancy and a ruptured ovarian cyst, a corpus luteum, or appendicitis. Blood tests and ultrasound can be used to differentiate these conditions. Blood tests did not help us in this case as her WBC was elevated. Serial HCG concentrations are not interpretable in the presence of both a viable intrauterine and ectopic pregnancy $(2,12,13)$.

The appearance on ultrasound of a tubal ectopic pregnancy varies considerably (2). When a normal intrauterine pregnancy is visualized on ultrasound, heterotopic pregnancy is usually not considered. This often makes diagnosis difficult. Secondly, as evident in our case a visualized intrauterine pregnancy decreases suspicion for ectopic pregnancy. Ultrasonography is the imaging modality of choice for pregnant women (2). It does not produce potentially harmful ionizing radiation, is readily available, and allows real-time imaging. Transvaginal ultrasound (TVUS) is the principle approach used for sonographic evaluation of pregnancy of unknown location. TVUS allows for earlier and more reliable detection of an intrauterine or ectopic pregnancy $(4,12)$. One study identified 388 ectopic pregnancies via trans-vaginal ultrasound. Sixty-four percent appeared inhomogeneous; 22\% had an empty gestational sac and $14 \%$ had a gestational sac containing a yolk sac or fetal pole (2). Approximately $26 \%$ of women will have absence of findings on sonography (5). In our case, a sonographer made a diagnosis of a fluid filled fallopian 
tube, which he discounted as nonpathological. Also presence of an ovarian cyst made visualization of an ectopic pregnancy difficult. The presence of intrauterine pregnancy significantly complicates the diagnosis of heterotopic pregnancy. The Transvaginal US image below demonstrates a complex right adnexa with two cysts. The thick walled cyst (marked by the solid white arrow) is separate from the right ovary (not shown) and lies medial to a larger thin walled internally septated anechoic cyst (marked by the blank arrow) which is most probably a right tubal ectopic (in this case of heterotopic pregnancy). The intrauterine gestational sac (marked by the solid white arrowhead) is also seen in this image.

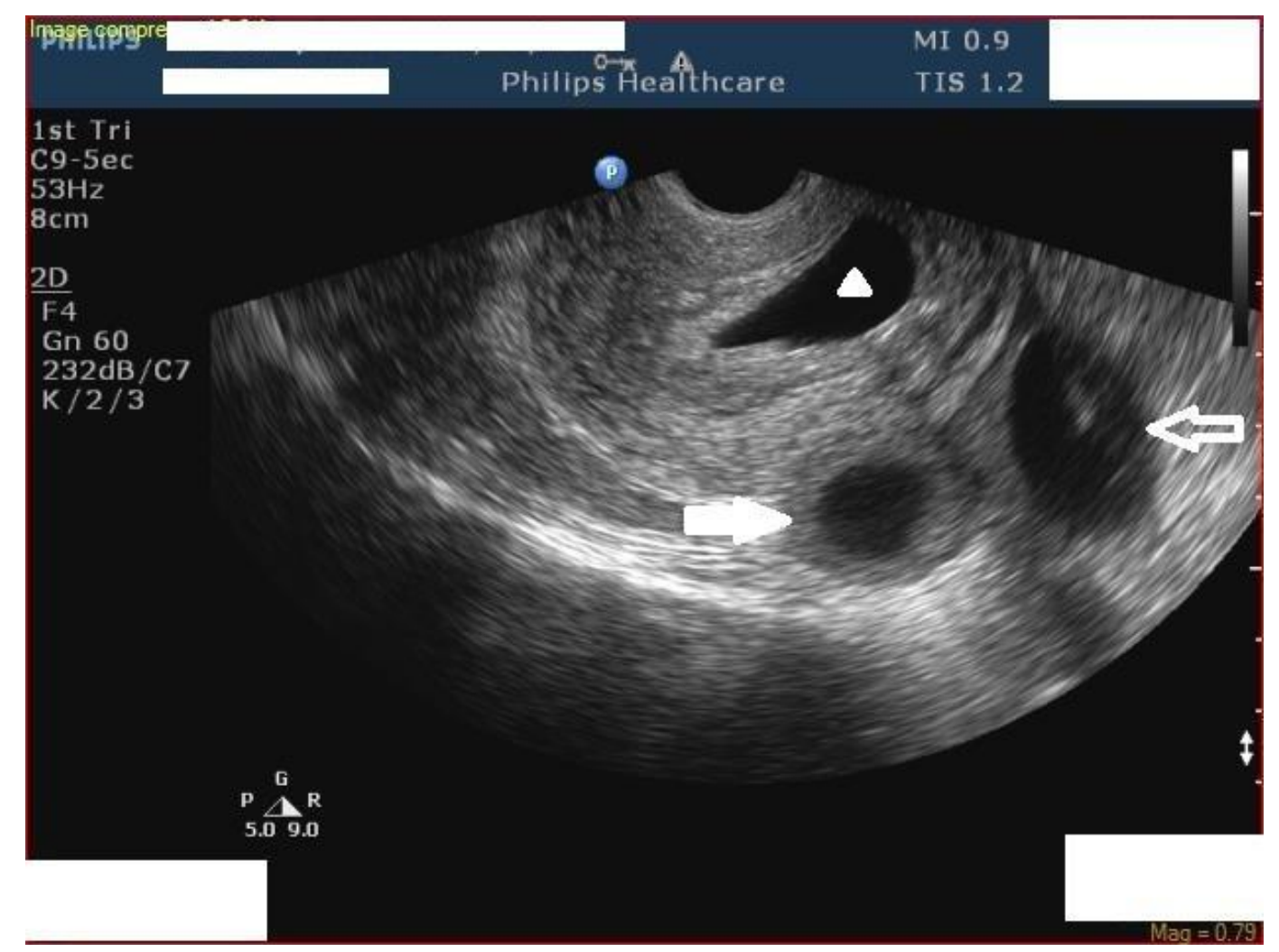

Fig 5 Transvaginal US demonstrates a complex right adnexa with two cysts. The thick walled cyst (marked by the solid white arrow) and internally septated anechoic cyst (marked by the blank arrow).

The abdominal examination is often unremarkable or may reveal lower abdominal tenderness. A complete pelvic examination should be performed. A bimanual pelvic examination is performed; the examination is often unremarkable in a woman with a small, unruptured ectopic pregnancy. The speculum examination is used to confirm that the uterus is the source and volume of bleeding. If rupture with significant bleeding has occurred, the abdomen may be distended and diffuse or localized tenderness to palpation and/or rebound tenderness may be found on examination. Women with hemodynamic instability and suspected ectopic pregnancy require emergency surgery.

The management of heterotopic pregnancy remains controversial. Systemic medical therapy (eg, 
methotrexate) is contraindicated in the presence of a viable intrauterine gestation $(3,7,12)$. However if expertise available and pregnancy has not ruptured, ultrasound guided local injection into the sac is effective with low toxicity to the concurrent intrauterine pregnancy treatment. The agents of choice should have high therapeutic effectiveness, and produce no lasting damage to the fallopian tube $(3,10,13)$. Two options are potassium chloride (KCL) and hyperosmolar glucose $(3,10,11,13)$. In extremely difficult cases such as cervical or caesarean section scar ectopic pregnancies, potassium chloride or a hyperosmolar solution are useful (12).

Salpingectomy is the standard surgical approach of a coexistent tubal pregnancy, and should be the first line of treatment in patients with hemodynamic instability or other signs of tubal rupture $(8,9,12)$. A laparoscopic approach is desirable if the patient is hemodynamically stable $(1,8,9,12)$. The Society of Gastrointestinal and Endoscopic surgeons recommend that laparoscopy versus laparotomy has the same decision algorithms as the non-gravid patient. The approach is based on the expertise of the operating surgeon and resources available. Laparoscopy can be performed during any trimester safely $(14,15)$. In our patient, we preformed single incision laparoscopy utilizing the Olympus system with an insufflation of $13 \mathrm{mmHg}$. Single-incision laparoscopy (SIL) is an evolving concept that has sent a tidal wave through the field of minimally invasive surgery. It utilizes the concept of inline viewing and a single incision that accommodates all of the working instruments. This procedure is technically more complex and time consuming. We were able to complete the entire procedure in about an hour and with no instrumentation of the gravid uterus.

\section{CONCLUSIONS}

Heterotropic pregnancy can be a potentially deadly condition. It is a rare entity that has a rising incidence as a result of increase use of reproductive technology. A high index of suspicion is required for their diagnosis. Transvaginal ultrasound remains the investigation of choice. Treatment modalities include surgical and non -sugircal methods and should be tailored according to the patient. Surgery remains the mainstay in the patient with evidence of rupture. 


\section{REFERENCES}

1. Eom J, Choi J, Hur C, et al. Surgical and obstetric outcomes of laparoscopic management for women with heterotopic pregnancy. The Journal Of Obstetrics And Gynaecology Research [serial online]. December 2013; 39(12):1580-1586.

2. Kirk E, Daemen A, Papageorghiou AT et al . Why are some ectopic pregnancies characterized as pregnancies of unknown location at the initial transvaginal ultrasound examination? Acta Obstetricia et Gynecologica Scandinavica 2008; $87: 1150$ - 1154 .

3. Ikechukwu, E., \& Adeleni, M. Heterotopic pregnancy with live infant. Annals Of African Medicine, 2013;12(1), 43-45. doi:10.4103/1596-3519.108251

4. Nyberg DA, Mack LA, Jeffrey RB Jr, Laing FC. Endovaginal sonographic evaluation of ectopic pregnancy: a prospective study. AJR Am J Roentgenol 1987; 149:1181.

5. Dogra V, Paspulati RM, Bhatt S. First trimester bleeding evaluation. Ultrasound Q 2005; 21:69.

6. Casanova BC, Sammel MD, Chittams J, et al. Prediction of outcome in women with symptomatic first-trimester pregnancy: focus on intrauterine rather than ectopic gestation. J Womens Health (Larchmt) 2009; 18:195.

7. Louis-Sylvestre C, Morice P, Chapron C, Dubuisson JB. The role of laparoscopy in the diagnosis and management of heterotopic pregnancies. Hum Reprod 1997; 12:1100.

\section{Barrenetxea $\mathrm{G}$, Barinaga-}

Rementeria L, Lopez de Larruzea A et al . Heterotopic pregnancy: two cases and a comparative review. Fertility and Sterility 2007; 87: 417.e9-e15 .

9. Goldstein JS, Ratts VS, Philpott T, Dahan MH. Risk of surgery after use of potassium chloride for treatment of tubal heterotopic pregnancy. Obstet Gynecol 2006; 107:506.

10. Goldberg JM, Bedaiwy MA. Transvaginal local injection of hyperosmolar glucose for the treatment of heterotopic pregnancies. Obstet Gynecol 2006; 107:509.

11. Salomon LJ, Fernandez H, Chauveaud A, et al. Successful management of a heterotopic Caesarean scar pregnancy: potassium chloride injection with preservation of the intrauterine gestation: case report. Hum Reprod 2003; 18:189.

12. Talbot K, Simpson R, Price $\mathrm{N}$ et al . Heterotopic pregnancy review. Journal of Obstetrics and Gynaecology 2011;31 : 7 12 .

13. Park HR, Moon MJ, Ahn EH (2009) Heterotopic quadruplet pregnancy: conservative management with ultrasonographically-guided $\mathrm{KCl}$ injection of cornual pregnancy and laparoscopic operation of tubal pregnancy. Fetal Diagn Ther 26:227-230

14. Rollins MD, Chan KJ, Price RR. Laparoscopy for appendicitis and cholelithiasis during pregnancy: a new standard of care. Surg Endosc 2003

15. Sages. Guidelines for Diagnosis, Treatment, and Use of Laparoscopy for Surgical Problems during Pregnancy 2011 